\title{
Release from competition and protection determine the outcome of plant interactions along a grazing gradient
}

\author{
Yoann Le Bagousse-Pinguet, Elisabeth M. Gross and Dietmar Straile \\ Y. Le Bagousse-Pinguet (y.b-pinguet@orange.fri), E. M. Gross and D. Straile, Limnological Inst., Univ. of Konstanz, Mainaustrasse 252, DE-78464 Konstanz, \\ Germany. Present address for YLBP: U.M.R. BIOGECO, Univ. of Bordeaux, Avenue des facultés, FR-33405 Talence, France.
}

\begin{abstract}
Plant interactions are suggested to shift from competition to facilitation and collapse with increasing grazing pressure. The existence of this full range of plant interactions and the role of underlying mechanisms (i.e. release from competition and protecting effect) in response to herbivory remains poorly documented and mainly described in terrestrial systems. We use a large grazing disturbance gradient (five levels of grazing) to test its effect on the outcome of plant interactions and underlying mechanisms in freshwater ecosystems. In a mesocosm experiment, we manipulated the presence of neighbouring plants to test their negative (competition) or protective (facilitation) effects on target plants along the grazing pressure gradient. We predicted that plant interactions 1) shift from competition to indirect facilitation with increased grazing pressure, 2) indirect facilitation collapses at high levels of grazing, 3) release from competition mainly drives the outcome in lowly grazed conditions and, 4) decreased protection occurs in highly grazed conditions responsible for the collapse of facilitation. This study shows the occurrence of the full range of outcomes in plant interactions under a wide spectrum of grazing pressure and indicates how the complex combination of underlying mechanisms shapes variations in plant interactions. We show that both, the release from competition and the increased protection by neighbouring plants drove the shift from competition to indirect facilitation. Declined protection by neighbouring plants resulted in a collapse of indirect facilitation for survival under intense herbivory. Our study provides the first experimental evidence of indirect facilitation structuring freshwater ecosystems thereby validating important ecological concepts mainly developed for terrestrial ecosystems.
\end{abstract}

Disturbance factors such as herbivory are critical components of land use impacts on plant communities over vast areas of the globe (Grime 1973, Cyr and Pace 1993, Gandhi and Herms 2010). Theoretical approaches suggested that grazing has not only direct effects but can be mediated via alteration of plant interactions (Bertness and Callaway 1994, Brooker and Callaghan 1998, Mulder and Ruess 1999, Bruno et al. 2003). Nonetheless, there is still little experimental evidence on how plant interactions can change the effect of grazing disturbance along large ecologically relevant gradients (but see Baraza et al. 2006, Smit et al. 2007, 2009). Understanding the variations and shifts in plant community responses at different levels of herbivory will increase our ability to predict community dynamics and ecosystem services of grazed systems, also under scenarios of global change (Post and Pedersen 2008).

The direction of plant interactions may shift along large gradients, with a dominance of competition in productive environments and facilitation under stressed or disturbed conditions (Bertness and Callaway 1994, Brooker and Callaghan 1998, Bruno et al. 2003). At low levels of grazing, herbivores can alleviate competitive exclusion and constraints on species establishment (i.e. release from competition, Grubb 1977). This may especially be important under productive conditions, where herbivores can prevent light competition by tall, dominant species (Grime 1973, Connell 1978). When grazing intensity increases, indirect facilitation can occur resulting from neighbouring plant protection, i.e. biotic refuges (physical barrier such as spine presence) or associational avoidances (reviewed by Milchunas and Noy-Meir 2002). Mechanisms of associational avoidance can be explained by 1) 'the repellent plant hypothesis' suggesting that grazing intolerant plants gain protections from surrounding grazing tolerant plants, and 2) 'the attractant decoy hypothesis' suggesting that a highly palatable plant may divert herbivores away from the less preferred plant. These positive effects of associational avoidances are likely to be mediated by the density of surrounding neighbours. Although both protection and release from competition have been well-documented and are assumed to be leading to shifts in the net outcome of plant-plant interactions, little is known on their co-occurrence along grazing disturbance gradients and how they interact to shape the outcomes of plant interactions.

The net outcome of plant interactions also depends on the chosen estimator of performance (Maestre et al. 2005, Brooker et al. 2008, Gomez-Aparicio et al. 2008), the natures of benefactors and beneficiary species (Baraza et al. 2006, Brooker et al. 2008, Gomez-Aparicio et al. 2008) and the length of the gradients under consideration (Maestre et al. 2005, Lortie and Callaway 2006, Brooker et al. 2008). This latter point may be crucial to explain the difference between the predictions 
of Bertness and Callaway's (1994) model (i.e. a shift from competition to facilitation with increasing abiotic and biotic constrains) and results of recent studies showing patterns of declines in facilitation (Kitzberger et al. 2000, Maestre and Cortina 2004). Using gradients with several levels of the manipulated factors may allow us to move beyond the clear limitations of previous two-level experimental studies and to clarify the relationship between plant interactions and disturbances (Brooker et al. 2008). Yet, few recent three-level experimental studies provided suitable advances by reporting the occurrence of indirect facilitation in intermediated grazedsystemsandacollapseunderhighbioticpressure(Brooker et al. 2006, Smit et al. 2007, Graff et al. 2007, Levenbach 2009, Vandenberghe et al. 2009). However, these studies did not report any significant competition at their lowest level of disturbance (but see Graff et al. 2007, Levenbach 2009). Low biotic disturbance resulted in net neutral effects of the spiny shrub Rosa rubiginosa on the performance (survival and height growth) of four species of tree seedlings (Smit et al. 2007, Vandenberghe et al. 2009). Thus, because of the low number of studies providing three- or more level-experimental designs and showing competition under low biotic disturbance, the role of both release from competition and protection on the relationship between plant interactions and disturbance remains unclear.

Aquatic ecosystems are well-known to be at least as much affected by biotic disturbance (i.e. grazing) than terrestrial ecosystems (Cyr and Pace 1993) and constitute interesting study systems to understand how plant interactions and underlying mechanisms (i.e. release from competition and protection) can drive plant communities. To our knowledge, prior studies only focused on changes in plant competition (Center et al. 2005), and no study examined shifts in plant interactions in freshwater environments. Submersed aquatic plants (commonly named 'macrophytes') play a key structuring role in freshwater ecosystems (Scheffer et al. 1993). Competition for nutrients among macrophyte species is usually strong, resulting in the local dominance of a few species (Gopal and Goel 1993). Their local dominance is also strongly dependent on grazing pressure (Sheldon 1987), and mechanisms of associational avoidance are likely to occur, at least in shoreline plants (Parker et al. 2007).

The main objectives of this manuscript are to investigate the responses of aquatic plant communities to disturbances in freshwater ecosystems along a five-level experimental gradient of grazing pressure. We predicted that plant interactions 1) shift from competition to indirect facilitation for both survival and biomass of target species with increased grazing pressure, 2) indirect facilitation collapses at high levels of grazing, 3) release from competition mainly drives the outcome in low grazed conditions and, 4) protection occurs in highly grazed conditions to drive the collapse of facilitation. To test these hypotheses, we set up a mesocosm experiment in two consecutive years (2008 and 2009). We chose three widespread submersed aquatic macrophytes with contrasting responses to grazing (Potamogeton perfoliatus, Potamogeton pectinatus and Myriophyllum spicatum) to study the outcome of plant interactions at five levels of grazing pressure.

\section{Material and methods}

\section{Study site}

The experiment was located at the Limnological Inst., Univ. of Konstanz, Germany (Konstanz, 47 $39^{\prime} 48 \mathrm{~N}, 9^{\circ} 10^{\prime} 31 \mathrm{E}$, and elevation $443 \mathrm{~m}$ a.s.I.). The climate is northern temperate. The plant growing season starts in early May, and finishes at the end of September (Miler 2008).

\section{Target species}

Submersed aquatic macrophytes are known to be a morphologically and functionally diverse group of species, for example in their use of space and resources in soil and water (Engelhardt and Richie 2002). We chose three species of submersed aquatic macrophytes: the monocots Potamogeton perfoliatus, Potamogeton pectinatus and the dicot Myriophyllum spicatum for this study. All are widely distributed in the northern hemisphere with different types of morphology and defence strategies (Gross et al. 2002). All can grow in monospecific or mixed stands (Engelhardt and Richie 2002). Potamogeton perfoliatus is a fast growing species producing dense canopies (Wolfer and Straile 2004) with high nutrient content and sensitivity to the larvae of Acentria ephemerella (Crambidae, Lepidoptera), a herbivorous moth (Gross et al. 2001, 2002, Miler and Straile 2010) and a high productivity to compensate biomass loss by herbivory (Sheldon 1987). Potamogeton perfoliatus is a dominant macrophyte species in many lakes including Lake Constance (Wolfer and Straile 2004, Sandsten and Klaassen 2008).

To measure the competitive or facilitative effect of $P$. perfoliatus stands (hence 'neighbours') under grazing disturbance, we selected three different target species: $P$. perfoliatus (to test intra-specific interactions) and M. spicatum and $P$. pectinatus (to test inter-specific interactions). Potamogeton pectinatus has a filiform leaf morphology, a high root-to-shoot ratio and is deeply rooted in the sediment (Engelhardt and Richie 2002). It is also highly sensitive to grazing pressure by A. ephemerella (Gross et al. 2002). Myriophyllum spicatum is a competitive species occurring in a large range of freshwater ecosystems in the northern hemisphere (Gross et al. 2001) producing defensive secondary metabolites (Smolders et al. 2000), which can affect insect herbivores such as A. ephemerella (Gross et al. 2001).

Acentria ephemerella is an herbivorous aquatic moth, which occurs in a wide range of freshwater ecosystem of the Northern Hemisphere (Gross et al 2001). Its larval stage is considered the major herbivore of macrophyte communities in Lake Constance (Miler and Straile 2010). Acentria's populations can strongly vary over the growing season (up to 50 individuals per gram of plant dry mass) in Lake Constance (Gross et al. 2002, Miler 2008).

\section{Matrix construction}

To test the competitive or facilitative effect of $P$. perfoliatus neighbours under grazing disturbance, monospecific stands (henceforth matrices) were established in May 2008 and May 2009 using apical shoots of established plants, reflecting the predominant vegetative propagation in perennial submersed aquatic plants. Apical shoots were collected in early May 2008 
and 2009 in Lake Constance. Six apical shoots of similar size (20 cm long) were randomly selected and planted in a regular pattern in $40 \times 90 \times 35 \mathrm{~cm}$ pots $(90 \mathrm{l}$ ) (Supplementary material Appendix A1a). This density corresponds to a shoot density observed at the beginning of the growing season in Lake Constance (Wolfer and Straile 2004). Bare soil plots were also installed in pots at this time in order to test the performance of isolated target species individuals (Supplementary material Appendix A1b). All the plots were independent from each other and received natural unsterilized sediment from Lake Constance. Plots were enclosed by transparent plastic foils in order to isolate sediment, water column and plants. In total, 15 matrices of $P$. perfoliatus were planted and 15 plots with bare soil were prepared for the experiment each year (2008 and 2009). All plots were placed in an outdoor mesocosm (mesocosm size: length $=10.5 \mathrm{~m}$, width $=5 \mathrm{~m}$, height $=1.5 \mathrm{~m}$ ) filled about $90 \mathrm{~cm}$ high with water from Lake Constance and were grown for 1.5 months to achieve sufficient biomass (mean \pm SE: biomass $=9.45 \pm 1.29 \mathrm{~g}$ ) to mimic the biomass of $P$. perfoliatus under field conditions (Wolfer and Straile 2004). Water temperature in the mesocosms showed seasonal dynamics similar to those in the lake's littoral zone, i.e. minimum temperatures during the experiment occurred in September $\left(14^{\circ} \mathrm{C}\right)$ and maximum ones in June $\left(23^{\circ} \mathrm{C}\right)$.

To test the grazing impact of $A$. ephemerella on the competitive or facilitative effect of $P$. perfoliatus, we performed a disturbance gradient using five levels of grazing $(0,1,5,10$ and 20 individuals per gram of plant dry mass (ind. $\mathrm{g}^{-1} \mathrm{dm}$ )) corresponding to the variability in populations observed in the field (Gross et al. 2002, Miler 2008). Acentria ephemerella pupae were sampled from different upper Lake Constance macrophyte patches in late June 2008 and 2009 in order to produce a first generation of larvae. Hatched larvae were introduced at the different grazing densities to the respective plots in July 2008 and 2009. In late July 2008 and 2009, new adults hatched and were caught in order to prevent reproduction. A second generation of first instar larvae was introduced in early August in a similar way in order to mimic two generations of herbivores.

\section{Experimental design}

Our experiment was fully factorial, lasted one growing season and was repeated in 2008 and 2009 (from 10 June to 21 September in 2008: 104 days and from 15 June to 21 September in 2009: 99 days) (see also Supplementary material Appendix A1c for the detailed experimental design). Target species responses (P. perfoliatus, P. pectinatus and M. spicatum) to grazing pressure (from 0 to 20 ind. $\mathrm{g}^{-1}$ plant dry mass) with or without $P$. perfoliatus neighbours were tested using three replicates per combination of treatment in each year.

Before planting, shoots of each target species were cut back to $5 \mathrm{~cm}$ in height. One individual of each target species (three targets per pot) was planted in 2008 and again in 2009 either within the matrices (with neighbours) or in the bare soil pots (without neighbour). We assumed that during the experiment, interactions among the individuals from the three target species that were transplanted inside the matrices were negligible due to their small biomass as compared to dense $P$. perfoliatus constituting the matrices themselves.
In total, 180 target shoots were planted ((one shoot $\times$ three species per pot cultivated without neighbours $X$ five levels of grazing pressure $X$ three replicates $X$ two years) + (one shoot $X$ three species per pot cultivated with neighbours $X$ five levels of grazing pressure $X$ three replicates $X$ two years)).

\section{Measurements and data analysis}

Survival and biomass of target species were recorded in late September 2008 and 2009. Plants were harvested and total biomasses were determined by drying them at $70^{\circ} \mathrm{C}$ for $72 \mathrm{~h}$ before weighing.

Survival and biomass of targets species were used to assess individual response to grazing disturbance and neighbour presence in both years. Data were analyzed using logistic regression models and analysis of covariance (ANCOVA) for survival and biomass respectively, with year, species and neighbour presence as main factors and grazing pressure as a continuous variable. Additionally, we included a quadratic variable (grazing $^{2}$ ) in statistical models to allow for non-linear responses of survival and biomasses along the grazing disturbance gradient. We performed an additional statistical analysis at low levels of grazing (at 0,1 and 5 ind. $\mathrm{g}^{-1} \mathrm{dm}$ ) in order to test significant effects of the neighbour treatment (i.e. the occurrence of competition) and possible effects of release from competition. Biomass data were log-transformed before analyses. We first examined the full models including all higher orders interactions and subsequently removed the non-significant interactions. All analyses were performed using R ver. 2.8.1.

\section{Results}

\section{Survival response of target species}

Grazing significantly decreased the overall survival of all three target species (Table 1, Fig. 1a) (see original data in Supplementary material Appendix A2 and the variations in response ratio ( $\ln R R_{\text {neighbours }}$ in Supplementary material Appendix A4a), while this response to grazing was speciesspecific. $M$. spicatum was least affected by increased grazing pressure whereas $P$. perfoliatus experienced a strong decline in survival. The effect of grazing on the survival of the three target species was mediated by the presence of neighbours $(p<0.001$, Table 1). The overall neighbour effect was higher in 2009 than 2008. In the presence of neighbours, grazing disturbance had an overall non-linear influence on survival as expressed by the significant interaction between neighbour and grazing ${ }^{2}$. At low grazing intensity, the presence of neighbours mediated the response of the three target species (Table 2, Fig. 1b). The presence of neighbours had an overall significant negative effect on the survival of the three target species (neighbour: $p=0.03$, see also Supplementary material Appendix A4a), indicating competition. Competition did not differ between the three target species (no significant species effect and interaction of species with other factors). When grazing pressure increased, the survival of the three target species decreased in absence of neighbours but increased in presence of neighbours (grazing $\times$ neighbour: $p=0.009$ ), illustrating an effect of release from competition (Fig. 1b). Indirect facilitation peaked at intermediate levels of grazing, 
Table 1. Results of the logistic regression model for the effects of target species, grazing, neighbours, grazing 2 , year and their significant interactions on the survival of target species. Note that all higher orders of non-significant interactions were subsequently removed.

\begin{tabular}{lrcc}
\hline & \multicolumn{3}{c}{ Survival of target species } \\
\cline { 2 - 4 } & DF & $\chi^{2}$ & $p$ \\
\hline Species & 2 & 0.34 & 0.844 \\
Grazing & 1 & 4.84 & $0.028^{*}$ \\
Neighbor & 1 & 9.34 & $0.002^{* *}$ \\
Grazing & 1 & 3.31 & $0.069\left(^{*}\right)$ \\
Year & 1 & 0.93 & 0.336 \\
Species $\times$ Grazing & 2 & 6.83 & $0.033^{*}$ \\
Grazing $\times$ Neighbor & 1 & 14.34 & $<0.001^{* * *}$ \\
Neighbor $\times$ Grazing & 1 & 10.43 & $0.0012^{* * *}$ \\
Neighbor $\times$ Year & 1 & 4.16 & $0.04^{*}$ \\
Error (model) & 168 & & \\
\hline
\end{tabular}

i.e. all target species had a higher survival with neighbours than without (in particular for $P$. perfoliatus and $P$. pectinatus) (Table 1, Fig. 1a and Supplementary material Appendix A4a: intermediate and high levels of grazing). This shift in survival mediated by neighbours along the grazing disturbance gradient was similar among target species as no significant interaction between species, grazing and neighbour was detected. Finally, at high levels of grazing, the survival of the three target species was also higher in the presence of neighbours but the benefits strongly declined.

\section{Biomass response of target species}

Grazing pressure negatively affected the biomass of the three target species (Table 3, Fig. 2) (see also original data in
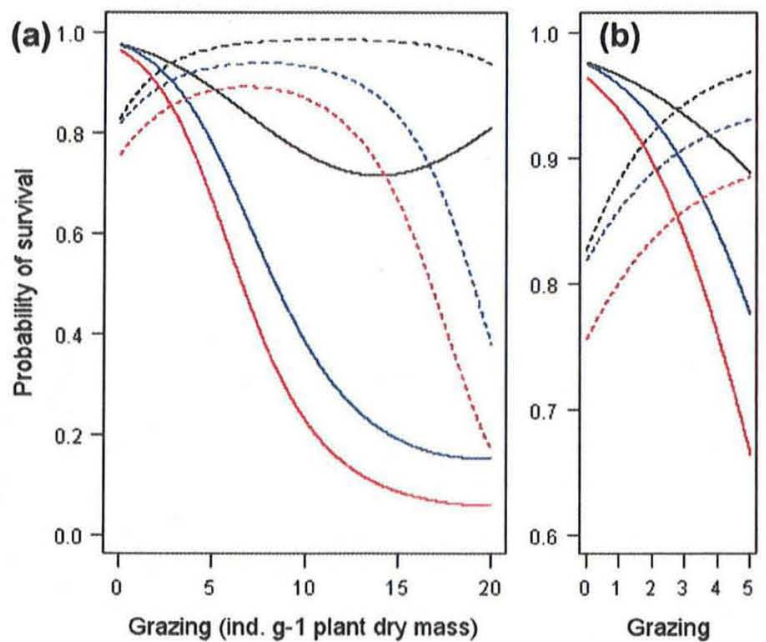

Figure 1. Predicted survival responses of the three target species (P. perfoliatus in red, $P$. pectinatus in blue, $M$. spicatum in black) along (a) the full grazing pressure gradient and (b) at low levels of grazing disturbance. Continuous lines represent the survival without neighbour and dotted lines the survival with neighbours. Predictions are based on our logistic regression models in Table 1 and 2 . Individual data points and standard errors of predictions are omitted to increase clarity of the figure, but are shown in Supplementray material Appendix A1.
Table 2. Results of the logistic regression model for the effects of target species, grazing, neighbours, year and their significant interactions on the survival of target species at low level of grazing disturbance. Note that higher order interactions have been subsequently removed when non-significant $(p>0.05)$

\begin{tabular}{lrcc}
\hline & \multicolumn{3}{c}{ Survival of target species } \\
\cline { 2 - 4 } & DF & $\chi^{2}$ & $p$ \\
\hline Species & 2 & 2.98 & 0.226 \\
Grazing & 1 & 5.36 & $0.021^{*}$ \\
Neighbor & 1 & 4.73 & $0.03^{*}$ \\
Year & 1 & 0.10 & 0.749 \\
Grazing $\times$ Neighbor & 1 & 6.78 & $0.009^{* *}$ \\
Error (model) & 101 & & \\
\hline
\end{tabular}

Supplementary material Appendix A3 and the variations in $\operatorname{lnRR}{ }_{\text {neighbours }}$ in Supplementary material Appendix A3b). As we observed for the survival, $M$. spicatum was least affected by grazing disturbance, $P$. pectinatus responded intermediately and $P$. perfoliatus was the most susceptible species. The presence of neighbours mediated the impact of grazing (Table 3 ). At low level of grazing, the presence of neighbours decreased the biomass of the three target species, indicating competition (Fig. 2, Supplementary material Appendix A4: left part). All target species benefited from the presence of neighbours (occurrence of facilitation) with increasing grazing pressure. We also detected a global year effect as the biomass of the target species was higher in 2009 (data not shown).

\section{Discussion}

Recent studies reported the occurrence of facilitation in intermediately grazed systems and its collapse under high biotic pressure (Brooker et al. 2006, Graff et al. 2007, Smit et al. 2007, Levenbach 2009, Vandenberghe et al. 2009). Here, we detected the full range of outcomes in plant interactions including significant competition at low grazing level by providing a five-level experimental gradient in a system primarily known for competition among plants. The occurrence of the full outcome points out how the complex combination of underlying mechanisms (i.e. the release from competition and protection) shapes variations in plant interactions. Both the release from competition and protection by neighbouring

Table 3. Results of the ANCOVA model for the effects of target species, grazing, neighbours, grazing2, year and their significant interactions on the biomass of target species. Note that all higher orders of nonsignificant interactions were subsequently removed.

\begin{tabular}{lrrc}
\hline & \multicolumn{3}{c}{ Biomass of target species } \\
\cline { 2 - 4 } & DF & F-ratio & $p$ \\
\hline Species & 2 & 81.34 & $<0.001^{* * *}$ \\
Grazing & 1 & 8.07 & $0.005^{* *}$ \\
Neighbor & 1 & 22.91 & $<0.001^{* * *}$ \\
Grazing & 1 & 0.48 & 0.489 \\
Year & 1 & 5.77 & $0.018^{*}$ \\
Species $\times$ Grazing & 2 & 3.42 & $0.036^{*}$ \\
Grazing $\times$ Neighbor & 1 & 36.50 & $<0.001^{* * *}$ \\
Error (model) & 127 & & \\
\hline
\end{tabular}




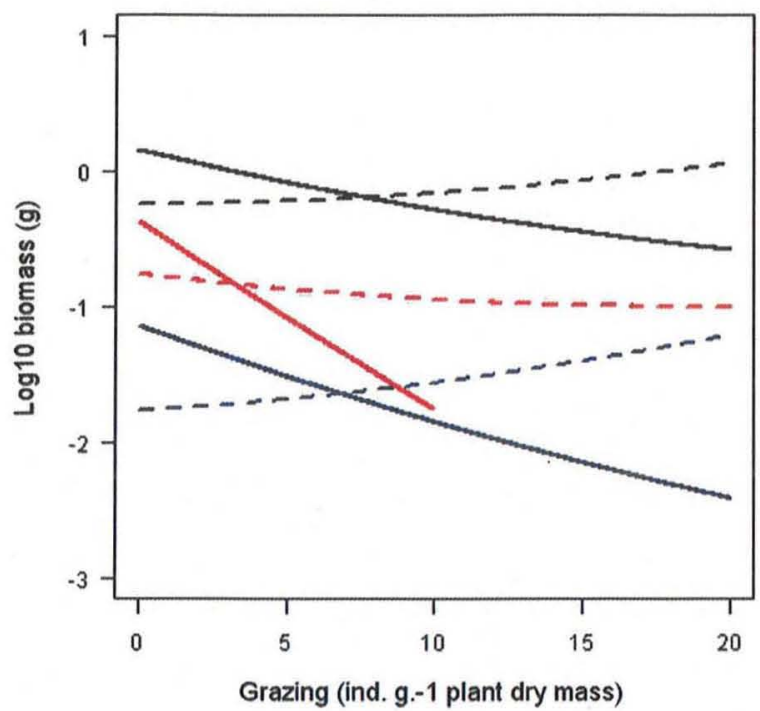

Figure 2. Predicted biomass responses of the three target species ( $P$. perfoliatus in red, P. pectinatus in blue, M. spicatum in black) along the full grazing pressure gradient. Continuous lines represent the survival without neighbour and dotted lines the survival with neighbours. Predictions are based on our ANCOVA model in Table 3 . Individual data points and standard errors of predictions are omitted to increase clarity of the figure, but are shown in Supplementary material Appendix A2.

plants led to a shift from competition to indirect facilitation as proposed by Bertness and Callaway (1994). Declined protection by neighbouring plants resulted in a collapse of indirect facilitation under intense herbivory. Thus, our results provide the first experimental evidence of indirect facilitation driving submersed freshwater plant communities. We validate important ecological concepts mainly developed for terrestrial (but see Levenbach 2009 for marine ecosystem) also for freshwater ecosystems, which are an understudied system for plant community theories, supporting the universality of these theories. Furthermore, one of the challenges of studying facilitation is reproducing stressful conditions in which positive interactions tend to occur. Greenhouse and controlled experiments typically place plants in idealized conditions where facilitation is unlikely to occur. We also demonstrate, for the first time, the occurrence of positive interactions in a controlled experiment (but see Espeland and Rice 2007 in a greenhouse experiment) highlighting a promising way to address further investigations on the role of facilitation for freshwater ecosystems.

\section{Relationship between plant interactions and grazing disturbance}

The three target species responded differently to grazing disturbance of Acentria ephemerella without neighbours for both survival and biomass in our experiment. The species-specific responses of the three target species to grazing are likely due to their differences in morphology and defence strategies (Engelhardt and Richie2002, Gross etal. 2002). For both survival and biomass, Myriophyllum spicatum was the most grazingtolerant species, Potamogeton pectinatus ranged intermediate and $P$. perfoliatus was the most susceptible. $M$. spicatum produces defensive phenolic secondary metabolites (Gross et al. 1996, Smolders et al. 2000), which affect herbivorous aquatic insects such as $A$. ephemerella (Choi et al. 2002, Gross et al. 2002). Conversely, P. pectinatus and $P$. perfoliatus are species that are strongly affected by the herbivory of A. ephemerella (Gross et al. 2002, Miler and Straile 2010). The intermediate response of $P$. pectinatus could be related to its filiform leaf morphology (Engelhardt and Richie 2002), conferring an advantage against herbivory compared to P. perfoliatus.

The presence of neighbours mediated the impact of grazing disturbance on target species performances both for the survival and biomass and led to indirect facilitation at intermediate disturbance levels. The presence of neighbours buffered the grazing pressure in a non-linear way for survival (Fig. 1a) and in a linear way for biomass (Fig. 2). According to several conceptual models (Bertness and Callaway 1994, Brooker and Callaghan 1998, Bruno et al. 2003), we observed a shift from competition to indirect facilitation among submersed aquatic plants from low to intermediate level of grazing disturbance. At very low level of grazing pressure or no grazing pressure, survival and biomasses of the three target species were always higher when growing without neighbour, underlining the occurrence of competition among submersed aquatic plants. Competition between macrophytes is usually strong (Gopal and Goel 1993). With increasing grazing pressure, the presence of neighbours maintained the performance (i.e. survival and biomass) of all the target species while it was collapsing without neighbours emphasizing the occurrence of indirect facilitation within these communities. Increasing disturbance from intermediate to high grazing levels led to a declined survival of the target species in the presence of neighbours, i.e. a collapse of indirect facilitation.

A collapse of indirect facilitation was, however, only revealed for survival whereas we only observed a shift from competition to facilitation for biomass. These results demonstrate that the selection of the estimator of plant performance has a strong influence on the net outcome of plant interactions (Maestre et al. 2005, Gomez-Aparicio et al. 2009), and that there is no reason to expect different aspects of plant performance to respond in a similar way towards facilitation and competition (Goldbergetal.1999, Hastwell and Facelli2003). Toconclude, our results stress the importance of using large gradients with several levels of the manipulated factors and the importance of the used estimator in order to detect the full range of variations in plant-plant interactions (i.e. the shift from competition to facilitation and a collapse at high level of constraint).

\section{Release from competition and protection shape the outcome of plant interactions}

The outcome of plant interactions along disturbance gradients is assumed to be shaped by both the release from competition and protection by neighbouring plants (Bertness and Callaway 1994). Our results illustrate the co-occurrence of both mechanisms regarding survival (Fig. 1). Survival of the three target species with neighbours benefited from increasing grazing pressure (Fig. 1b). Increased survival with increasing herbivory in the presence of neighbours was likely due to allcviation of neighbour compctitive effects (Grubb 1977). This mechanism may be important under productive 
conditions, whereherbivorycan mitigatedensitysuppression and competition by dominant plant species (Grime 1973, Connell 1978). With increasing grazing pressure from low to intermediate levels, survival of the three target species without neighbours dropped dramatically whereas the presence of neighbours maintained high survival (Fig. 1a). In accordance with other studies (Bertness and Callaway 1994, Milchunas and Noy-Meir 2002), we observed an increased protection by neighbours thereby preventing the negative effects of increased grazing disturbance. Finally, survival under intense herbivory pressure also collapsed with neighbours because of the declined benefactor effects (Michalet et al. 2006, Graffetal. 2007, Smit et al. 2009). Although we were able to observe that the release from competition and a protection by neighbours shaped the outcome of plant interactions, it was not possible to disentangle the relative importance of these two mechanisms along our grazing pressure gradient. It is likely that they co-occur over the full grazing gradient with a stronger importance of release from competition in productive environments and protection (indirect facilitation) under harsher conditions (Brooker et al. 2006, Michalet et al. 2006).

In our study, the most grazing-intolerant species ( $P$. perfoliatus) was used as neighbour because of its strong dominance in freshwater ecosystems (Wolfer and Straile 2004, Sandsten and Klaassen 2008). The observed benefits of neighbouring plants on target species suggest the occurrence of indirect facilitation by associational avoidance (sensu Milchunas and Noy-Meir 2002) and confirm the 'attractantdecoy hypothesis' proposing that highly palatable plants may divertherbivores away from theless preferred plants. The degree of protection offered by neighbour species actually declines with their increased palatability (Rousset and Lepart 2000, Baraza et al. 2006). When the neighbour is palatable, the result of the association can shift to greater consumption or damage of the plant species growing nearby (Baraza et al. 2006), termed 'apparent competition' (Connell 1990). The less palatable M. spicatum in our experiment might have a stronger protecting effect (sensu Baraza et al. 2006) or may have exacerbated the net outcome of competition. Since the focus of our study was not on herbivore foraging decision, we propose that further experiments manipulating neighbour palatability along large grazing disturbance gradients need to be performed in order to understand how herbivore foraging decision shapes the outcome of plant interactions.

This study experimentally demonstrated the importance of large gradients in order to observe the full variation in outcomes of plant interactions along a grazing disturbance gradient. We also underlined how mechanisms such as release from competition and protection shape the outcome of plant interactions. Finally, our study suggests that we may not only consider competitive interactions between macrophytes as previously done, but should have a stronger focus on positive interactions in order to understand all possible factors determining aquatic plant community structure and improve our knowledge on community ecology theories.

Acknowledgements - This research project was part of the Collaborative Research Centre (CRC) no. 454 'Littoral of Lake Constance' and was financially supported by the Deutsche Forschungsgemeinschaft
(DFG). We are grateful to N. Gross, P. Liancourt, R. Michalet and C. Smit for valuable comments.

\section{References}

Barraza, E. et al. 2006. Conditional outcomes in plant-herbivore interactions: neighbours matter. - Oikos 113: 148-156.

Bertness, M. D. and Callaway, R. M. 1994. Positive interactions in communities. - Trends Ecol. Evol. 9: 191-193.

Brooker, R. W. and Callaghan, T. V. 1998. The balance between positive and negative plant interactions and relationship to environmental gradients: a model. - Oikos 81: 196-201.

Brooker, R. W. et al. 2006. Transient facilitative effects of heather on Scots pine along a grazing disturbance gradient in Scottish moorland. - J. Ecol. 94: 637-645.

Brooker, R. W. et al. 2008. Facilitation in plant communities: the past, the present and the future. - J. Ecol. 96: 18-24.

Bruno, J. F. et al. 2003. Inclusion of facilitation in to ecological theory. - Trends Ecol. Evol. 18: 119-125.

Center, T. D. et al. 2005. Herbivory alters competitive interactions between two invasive aquatic plants. - Biol. Control 33: 173-185.

Choi, C. et al. 2002. Impact of polyphenols on growth of the aquatic herbivore Acentria ephemerella. - J. Chem. Ecol. 28: 2245-2256.

Connell, J. H. 1978. Diversity in tropical rain forests and coral reefs. - Science 199: 1302-1310.

Connell, J. H. 1990. Apparent versus "real" competition in plants. - In: Grace, J. B. and Tilman, D. (eds), Perspective in plant competition. Academic Press, p. 9-26.

Cyr, H. and Pace, M. L. 1993. Magnitude and patterns of herbivory in aquatic and terrestrial ecosystems. - Nature 361: $148-150$.

Engelhardt, K. A. M. and Richic, M. E. 2002. The effect of aquatic plant species richness on wetland ecosystem processes. - Ecology 83: 2911-2924.

Espeland, E. K. and Rice, K. J. 2007. Facilitation across stress gradients: the importance of local adaptation. - Ecology 88: 2404-2409.

Gandhi, K. J. K. and Herms, D. A. 2010. Direct and indirect effects of alien insect herbivores on ecological processes and interactions in forests of eastern northern America. - Biol. Invas. 12: $389-405$.

Goldberg, D. E et al. 1999. Empirical approaches to quantifying interaction intensity: competition and facilitation along productivity gradients. - Ecology 80: 1118-1131.

Gomez-Aparicio, L. et al. 2008. Facilitation of three saplings by nurse plants: microhabitat amelioration or protection against herbivores? - J. Veg. Sci. 19: 161-172.

Gopal, B. and Goel, U. 1993. Competition and allelopathy in aquatic plant communities. - Bot. Rev. 59: 155-210.

Graff, P. et al. 2007. Shifts in positive and negative plant interactions along a grazing intensity gradient. - Ecology 88: 188-199.

Grime, J. P. 1973. Competitive exclusion in herbaceous vegetation. Nature 242: 344-347.

Gross, E. M. et al. 2001. Experimental evidence for changes in submersed macrophyte species composition caused by the herbivore Acentria ephemerella (Lepidoptera). - Oecologia 127: 105-114.

Gross, E. M. et al. 2002. High abundance of herbivorous Lepidoptera larvae (Acentria ephemerella Denis \& Schiffermüller) on submerged macrophytes in Lake Constance (Germany). - Arch. Hydrobiol. 15: 1-27.

Grubb P. J. 1977. The maintenance of species richness in plant communities: the importance of the regeneration niche. - Biol Rev. 52: 107-145

Hastwell, G. T. and Facelli, J. M. 2003. Differing effects of shadeinduced facilitation on growth and survival during the establishment of a chenopod shrub. - J. Ecol. 91: 941-950. 
Kitzberger, T. et al. 2000. Effects of climatic variability on facilitation of tree establishment in northern Patagonia. - Ecology 81: 1914-1924.

Levenbach, S. 2009. Grazing intensity influences the strength of an associational refuge on temperate reefs. - Oecologia 159: 181-190.

Lortie, C. J. and Callaway, R. M. 2006. Re-analysis of meta-analysis: support for the stress gradient hypothesis. - J. Ecol. 94: 7-16

Maestre, F. T. and Cortina, J. 2004. Do positive interactions increase with abiotic stress? A test from a semi-arid steppe. Proc. R. Soc. B 271: 331-333.

Maestre, F. T. et al. 2005. Is the change of plant-plant interactions with abiotic stress predictable? A meta-analysis of field results in arid environments. - J. Ecol. 93: 748-757.

Michalet, R. et al. 2006. Do biotic interactions shape both sides of the humped-back model of species richness in plant communities? - Ecol. Lett. 9: 767-773.

Milchunas, D. G. and Noy-Meir, I. 2002. Grazing refuges, external avoidance of herbivory and plant diversity. - Oikos 99: 113-130.

Miler, O. 2008. The aquatic moth Acentria ephemerella as a key species in submerged aquatic vegetation - direct and trait-mediated interactions with predators and food plants. - PhD thesis, Univ. of Konstanz, Germany.

Miler, O. and Straile, D. 2010. How to cope with a superior enemy? Plant defence strategies in response to annual herbivore outbreaks. - J. Ecol. 98: 900-907.

Mulder, C. P. H. and Ruess, R. W. 1999. Effects of herbivory on arrowgrass: interactions between geese, neighbouring plants, and abiotic factors. - Ecol. Monogr. 68: 275-293.

Parker, J. D. et al. 2007. Beaver herbivory on aquatic plants. Oecologia 151: 616-625.
Post, E. and Pedersen, C. 2008. Opposing plant community responses to warming with and without herbivores. - Proc. Natl Acad. Sci. USA 105: 12353-12358.

Rousset, O. and Lepart, J. 2000. Positive and negative interactions at different life stages of a colonizing species (Quercus humilis). - J. Ecol. 88: 401-412.

Sandsten, H. and Klaassen, M. 2008. Swan foraging shapes spatial distribution of two submersed plants, favouring the preferred prey species. - Oecologia 156: 569-576.

Scheffer, M. et al. 1993. Alternative equilibria in shallow lakes. Trends Ecol. Evol. 8: 275-279.

Sheldon, S. P. 1987. The effects of herbivorous snails on submerged macrophytes communities in Minnesota lakes. - Ecology 68: 1920-1931.

Smit, C. et al. 2007. Nurse plants, tree saplings and grazing pressure: changes in facilitation along a biotic environmental gradient. - Oecologia 152: 265-273.

Smit, C. et al. 2009. Inclusion of biotic stress (consumer pressure) alters predictions from the Stress gradient hypothesis. - J. Ecol. 97: 1215-1219.

Smolders, A. J. P. et al. 2000. Phenolic contents of submerged, emergent and floating leaves of aquatic and semi-aquatic macrophyte species: why do they differ? - Oikos 91: 307-310.

Vandenberghe, C. et al. 2009. Does the strength of facilitation by nurse shrubs depend on grazing resistance of tree saplings? Basic Appl. Ecol. 10: 427-436.

Wolfer, S. and Straile, D. 2004. Spatio-temporal dynamics and plasticity of clonal architecture in Potamogeton perfoliatus. - Aquat. Bot. 78: 307-318 